\title{
Volume reduction of the jugular foramina in Cavalier King Charles Spaniels with syringomyelia
}

\author{
Martin Jürgen Schmidt ${ }^{1 *}$, Nele Ondreka ${ }^{1}$, Maren Sauerbrey ${ }^{1}$, Holger Andreas Volk ${ }^{3}$, Christoph Rummel ${ }^{2}$ \\ and Martin Kramer ${ }^{1}$
}

\begin{abstract}
Background: Understanding the pathogenesis of the chiari-like malformation in the Cavalier King Charles Spaniel (CKCS) is incomplete, and current hypotheses do not fully explain the development of syringomyelia (SM) in the spinal cords of affected dogs. This study investigates an unconventional pathogenetic theory for the development of cerebrospinal fluid (CSF) pressure waves in the subarachnoid space in CKCS with SM, by analogy with human diseases. In children with achondroplasia the shortening of the skull base can lead to a narrowing of the jugular foramina (JF) between the cranial base synchondroses. This in turn has been reported to cause a congestion of the major venous outflow tracts of the skull and consequently to an increase in the intracranial pressure (ICP). Amongst brachycephalic dog breeds the CKCS has been identified as having an extremely short and wide braincase. A stenosis of the JF and a consequential vascular compromise in this opening could contribute to venous hypertension, raising ICP and causing CSF jets in the spinal subarachnoid space of the CKCS. In this study, JF volumes in CKCSs with and without SM were compared to assess a possible role of this pathologic mechanism in the development of SM in this breed.

Results: Computed tomography (CT) scans of 40 CKCSs $>4$ years of age were used to create three-dimensional (3D) models of the skull and the JF. Weight matched groups (7-10 kg) of 20 CKCSs with SM and 20 CKCSs without SM were compared. CKCSs without SM presented significantly larger JF -volumes (median left JF: $0.0633 \mathrm{~cm}^{3}$; median right JF: $0.0703 \mathrm{~cm}^{3} ; \mathrm{p}<0.0001$ ) when compared with CKCSs with SM (median left JF: $0.0382 \mathrm{~cm}^{3}$; median right JF: $0.0434 \mathrm{~cm}^{3} ; \mathrm{p}$ $<0.0001)$. There was no significant difference between the left and right JF within each group. Bland-Altman analysis revealed excellent reproducibility of all volume measurements.
\end{abstract}

Conclusion: A stenosis of the JF and consecutive venous congestion may explain the aetiology of CSF pressure waves in the subarachnoid space, independent of cerebellar herniation, as an additional pathogenetic factor for the development of SM in this breed.

Keywords: Achondroplasia, Chiari-like malformation, Caudal occipital malformation

\section{Background}

Extensive studies have been carried out to explain a possible relationship between cranial and cervical dimensions and the development of the chiari-like malformation (CLM) and syringomyelia (SM) in the Cavalier King Charles Spaniel (CKCS). In an effort to identify pathogenetic alterations of the skull in this possible canine analogue to the human chiari malformation type 1 , work has focused on a mismatch of caudal fossa capacity and its contents as hypothesized in man [1-5]. Results of studies

\footnotetext{
* Correspondence: Martin.J.Schmidt@vetmed.uni-giessen.de

1 Department of Veterinary Clinical Sciences, Small Animal Clinic

Justus-Liebig-University, Frankfurter Straße 108, 35392 Giessen, Germany

Full list of author information is available at the end of the article
}

concerning an underdevelopment of the volume of the caudal fossa in these dogs were controversial, as smaller as well as normal volumes have both been found in CKCSs with $\mathrm{SM}$ in comparison to unaffected dogs $[1,3,6,7]$. The concept of overcrowding of the caudal skull compartment is not entirely satisfactory, as it cannot explain the absence of SM in cases with severe herniations or the presence of SM with minimal or absent herniation (also referred to as Chiari type 0 ). Neither the volume of the caudal fossa nor the severity of cerebellar herniation can predict the occurrence of SM [3,4]. However, it is generally accepted for the CKCS that $\mathrm{SM}$ is a consequence of abnormal cerebrospinal fluid flow in the foramen magnum and/or the upper spinal cord.

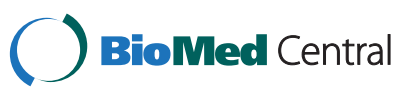

(c) 2012 Schmidt et al.; licensee BioMed Central Ltd. This is an Open Access article distributed under the terms of the Creative Commons Attribution License (http://creativecommons.org/licenses/by/2.0), which permits unrestricted use, distribution, and reproduction in any medium, provided the original work is properly cited. 
Turbulent flow and jets of CSF seem to be associated with the development of SM, and CSF flow velocity was related to the presence of SM in the CKCS $[2,5]$.

In the human literature there is debate as to whether obliteration of the foramen magnum is the only pathogenetic factor responsible for the development of CSF pressure waves, because herniation of less than $5 \mathrm{~mm}$ or even absent herniation can still be accompanied by classic Chiari symptomatology [8-10].

More than one or even multiple factors might be etiologically important and should be considered in the pathogenesis of CLM/SM in the CKCS.

Recently the CKCS has been shown to have an extremely high braincase length-width ratio (cranial index) compared to other brachycephalic dogs [11]. As only brachycephalic breeds seem to suffer from CLM [12], it would seem reasonable that the pathophysiological factors leading to this disease could somehow be associated with this severe brachycephaly, as has already been suggested for the Griffon Bruxellois [13]. A link between brachycephaly and abnormal CSF flow can be found in the skull base:

The longitudinal extension of the whole braincase is strongly dependent on the growth of the skull base [14]. The synchondrosis sphenooccipitalis and sphenopresphenoidalis (or intersphenoidalis) have been assumed to undergo a premature closure in brachycephalic dogs [15], as in humans suffering from achondroplasia [16]. In both disease entities enchondral ossification and growth are impaired in extremity- as well as skull base bones $[15,16]$. Although affected humans can be clinically normal, intracranial pressure (ICP) can be raised, cerebral fluid flow may be altered and ventriculomegaly and hydrocephalus $[17,18]$ may be detected. Stenosis of the jugular foramen (JF) leading to venous congestion has been suggested to be a consequence of the impaired longitudinal extension of the skull base. Congestion of the vascular compartment in the skull leads to a raised intracranial pressure $[17,19,20]$. A stenosis of the JF and a consecutive vascular compromise in this opening could contribute to venous hypertension, raised intraventricular and intracranial pressure, and significant CSF jets within the skull of the CKC too. This could eventually result in SM. It has been suggested this could be a contributory mechanism to the development of SM in Griffon Bruxellois that had no evidence of CM [13]. We hypothesize that the volume of the JF is reduced in CKCSs with SM. The aim of this study was therefore to compare the volumes of the JF in CKCSs affected with SM and CKCSs without SM in order to identify possible factors for the development of SM unrelated to cerebellar herniation.

\section{Methods}

As determinations of the diameter of skull foramina can be variable depending on the imaging plane [21] we decided to measure the volumes of the JF. These were determined using computed tomography (CT) data from the heads of 40 CKCS. The CT data sets were acquired with a multislice CT (CT Brilliance, Philips, Hamburg, Germany; $120 \mathrm{kV}, 350 \mathrm{mAs}$, matrix 512 x 512, slice thickness $0.8 \mathrm{~mm}$, pitch: $1 \mathrm{~mm}$ ).

Magnetic Resonance (MR)-images were used to identify $\mathrm{SM}$ in the cervical spinal cord (Gyroscan Intera, 1.0 Tesla, Philips, Hamburg, Germany). T1 and T2 weighted sagittal and transverse images of the head were obtained. T2 weighted and FLAIR sagittal images of the spinal cord up to the level of the fifth thoracic vertebra were acquired as well as a transverse fast field echo (FFE)-sequence of the spinal cord that was added if SM was present. All imaging studies were performed for breeding selection on the owner`s request. All dogs showed cerebellar indentation or deviation into the foramen magnum and obliteration of the CSF space around the caudal contour of the cerebellum (CLM). None of the dogs had a history of scratching or cervical hyperaesthesia; however, this was not an inclusion criterion. Dogs were selected according to the following criteria: Only adult dogs were chosen, as skull growth is incomplete in dogs $<1$ year [22]. As SM can be late in onset, only CKCSs 4 years or older were included in the study [23,24]. 20 CKCSs (7 male, 13 female) with SM (group 1) were compared with a control group of 20 CKCSs without SM (8 male, 12 female; group 2). Only CKCSs with a body weight between 7-10 $\mathrm{kg}$ were included to minimize allometric effects on the volume differences.

Image processing for volume determination of the JF was with done using graphical software (Amira ${ }^{\circledR}$ Graphical Software, Mercury Computer Systems, Berlin, Germany). The internal boundaries of the JF were segmented using hand tracing slice by slice in sagittal, dorsal and transverse CT images (Figure 1A, red area). Segmentation in this context describes the manual creation of an image mask. All voxels corresponding to a single anatomical structure in the images, e.g. the JF, are selected and assigned the same value in the mask. The final mask thus contains information about all the selected anatomical structures, and in combination with the original CT data and polygonal surface reconstruction algorithms allows the calculation of a specific volume in the dataset (Figure 1B). The dorsal and ventral boundaries of the JF were defined as follows:

The dorsal boundary was set along a line following the straight contour of the basioccipital bone from its midline laterally. The ventral boundary was set along a line perpendicular to the first line through the ventro-medial margin of the JF (Figure 1C). Although this line might not represent the true ventro-lateral margin of the JF, this method helped to define this boundary in an objective manner in all dogs. 

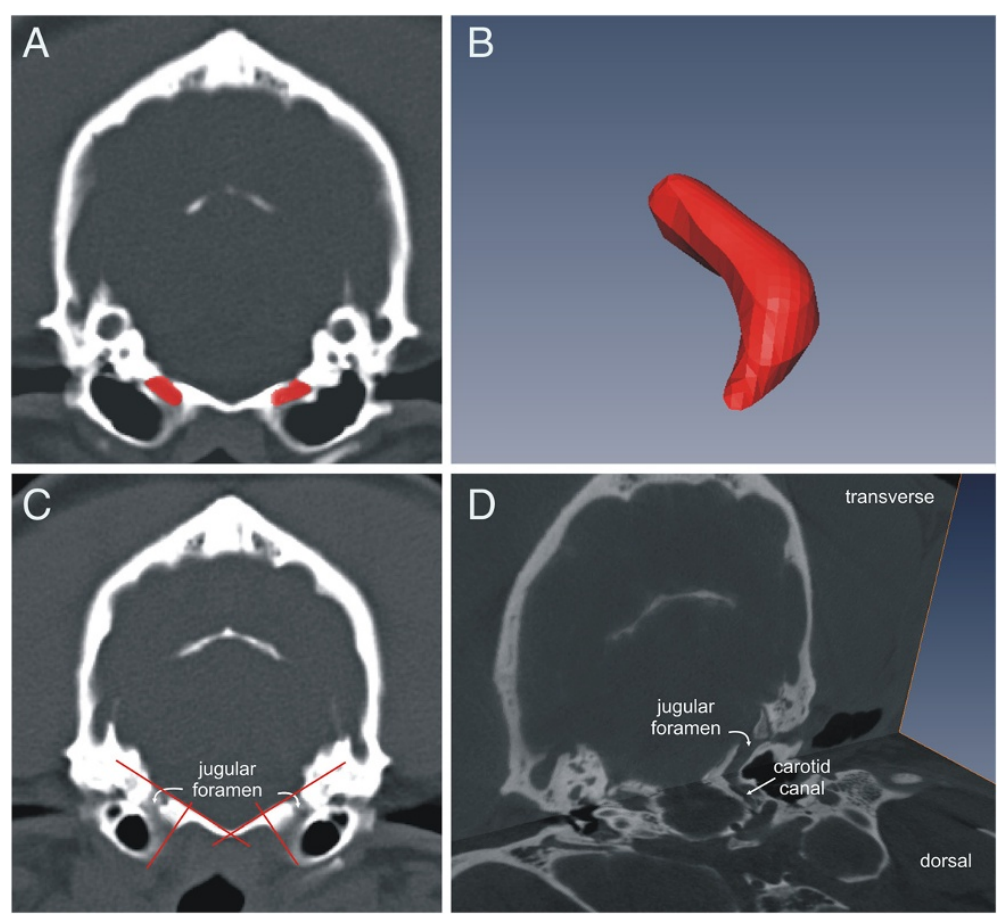

Figure 1 Transverse CT image of the manual segmentation process of the area of the jugular foramen. Outlines of the bony boundaries of the JF were manually segmented and assigned to tissue label fields $(\mathbf{A})$. The segmented areas are reconstructed to the jugular foramen volume (B). The dorsal boundary of the JF was set along a line following the straight contour of the basioccipital bone from its midline laterally. The ventral boundary was set along a line perpendicular to the first line through the ventro-medial margin of the JF (C). Multiplanar image reconstruction (D) helped to identify the internal boundaries of the JF.

Multiplanar image reconstruction (Figure $1 \mathrm{D})$ and volume rendering (Figure 2) helped to identify the internal boundaries of the JF. The differentiation of anatomical structures within close proximity to the JF, in particular the carotid canal, whose lumen was in contact with the JF in some dogs, was not always possible by examining a single plane.

Volumes of left and right JFs were determined for CKCSs with and without SM and compared. Volume determinations were carried out blinded to the MRI

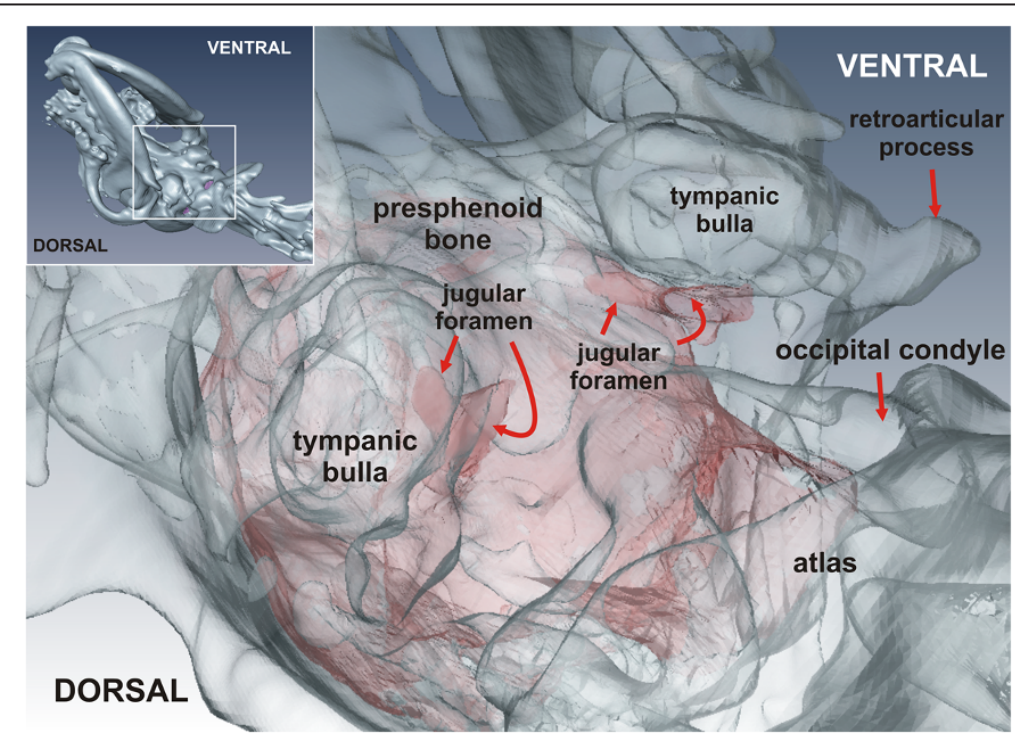

Figure 2 Reconstruction of the skull and jugular foramen. Oblique ventral view of a volumetric reconstruction of the skull (transparent) containing the JF of both sides (red). The reconstruction shows the channel-like conformation of the JF. 
findings and the order of the datasets was randomised. All volume determinations were performed twice by the same examiner (MS) in two different sittings.

\section{Statistical analysis}

All data was analysed using a statistical program package (Graph Pad Prism 4.0, Graph Pad Software Inc., and San Diego, California). Data was analysed for normality using a Kolmogorow-Smirnov test.

We tested for significant differences in left and right JF volumes between group 1 and group 2 using a student's t-test (Figure 3A/C). As variations between the left and right JF of dogs have been reported [25], a possible difference between the left and right JFs within groups 1 and 2 was also evaluated using the same test.

Furthermore, to assess the precision of the manual segmentation process and the determined volumes, the reproducibility of the volume calculation was measured using a Bland-Altman analysis, which compares the differences between the first and second volume measurement of each dog. The differences between the two measurements were then plotted against the average (mean) of the two measurements. In the graphic presentation of this analysis (Figure 4 A-D), a full horizontal line was drawn at the mean difference, and dotted lines were drawn at the limits of agreement, which were defined as the mean difference plus or minus twice the standard deviation (SD) of the differences. Reproducibility is considered good if $95 \%$ of the differences are within these two standard deviations (gray field between the dotted lines).

Thereafter, a student's t-test was again used to establish if there were statistically significant differences between the groups using the data of the second volume determination (Figure 3B/D). P-values less than 0.05 were considered to be statistically significant.

\section{Results}

The volumes of the JF could be clearly determined. Figure 2 shows an example of the 3D skull models and the JFs.

Comparisons between JF volumes from the first measurement are presented in Figure 3A-C. The median left JF volume of CKCSs without SM was $0.063 \mathrm{~cm}^{3}$, and the median right JF volume of CKCSs without SM was $0.07 \mathrm{~cm}^{3}$. The median left JF volume of CKCSs with SM was $0.038 \mathrm{~cm}^{3}$, and the median right JF volume of CKCSs with SM was $0.043 \mathrm{~cm}^{3}$. Comparing the volumes of both the left and right JF of the CKCSs with SM to the CKCSs without SM revealed a clear statistically significant difference with $\mathrm{p}<0.0001$.

There was no significant difference between the volumes of the left and right JF within either group $1(\mathrm{p}=$ $0.145)$ or group $2(\mathrm{p}=0.084)$.
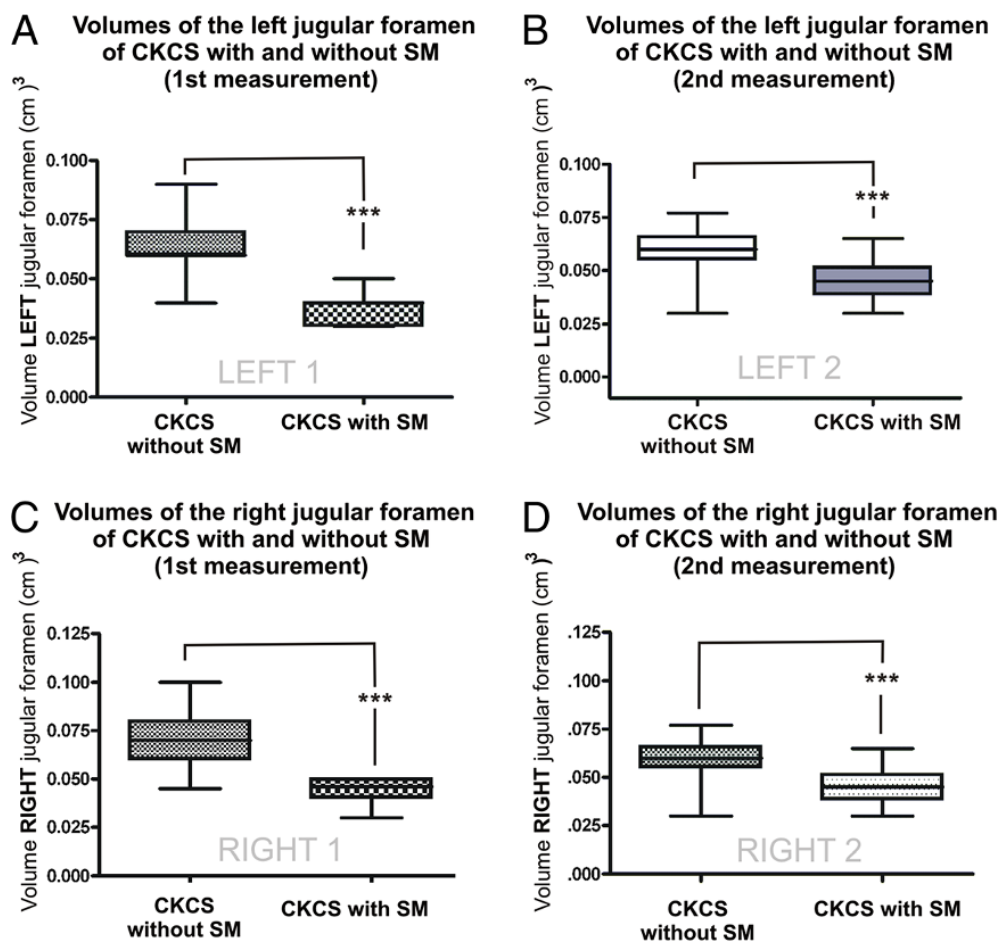

Figure 3 Graphical representation of the comparison of jugular foramen (JF) volumes in Cavalier King Charles Spaniels with and without syringomyelia (SM). Figure 3A presents differences in the volume of the left JF between CKCSs with and without SM. Figure 3B shows the comparison of the same volumes collected from a second measurement. Figure $3 \mathbf{C}$ presents a comparison of the volume of the right JF between CKCSs with and without SM. Figure 3C present the results of the same volumes collected in a second measurement. 

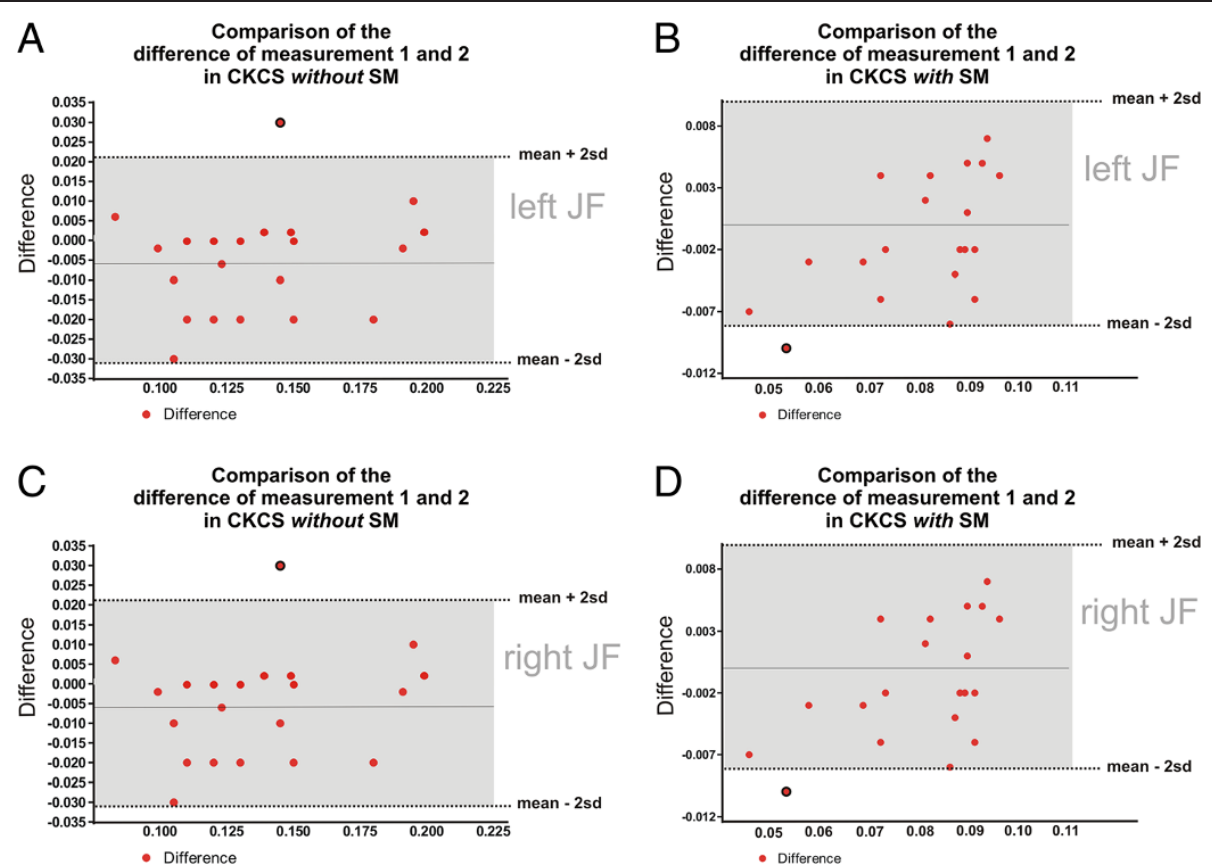

Figure 4 Graphical presentation of comparison of two measurements of the left and right jugular foramina in Bland-Altman plots. The reproducibility of two segmentation processes is demonstrated. The differences between the two measurements (red dots) are plotted against the averages of the differences. Dotted lines indicate the lower and upper limits of agreement (mean difference $\pm 2 x$ standard deviations). $95 \%$ of all differences are within two standard deviations, representing excellent reproducibility. Outliers are marked with a black rim.

The results from the second measurement of left and right JF volumes are presented in Figure 3-D. The median left JF volume of CKCSs without SM was $0.059 \mathrm{~cm}^{3}$, and the median right JF volume of CKCSs without SM was $0.07 \mathrm{~cm}^{3}$. The median left JF volume of CKCSs with SM was $0.045 \mathrm{~cm}^{3}$, and the median right JF volume of CKCSs with SM was $0.044 \mathrm{~cm}^{3}$. Comparing the volumes of both the left and right JF of CKCSs with SM to CKCSs without SM again demonstrated a clear statistically significant difference, with $\mathrm{p}<0.0001$.

Also in the second measurement there was no significant difference between the left and right JF volumes within either group $1(\mathrm{p}=0.12)$ or group $2(\mathrm{p}=0.096)$.

The assessment of reproducibility is shown in Figure 4 A-D. Ninety-five percent of the differences between the first and second volume determinations were less than \pm 2 $\mathrm{SD}$ from the mean difference. The mean difference in Figure 3 A-D was negative, indicating a tendency to measure a larger volume for the JFs in the second calculation. In comparing differences between the first and second measurements of the left JF of CKCSs with SM the mean difference was positive, indicating a tendency to measure a smaller volume in the second calculation in these animals (Figure 3B).

These figures indicate there was little variation between measurements. The data shows that both the left and right JF of CKCSs with SM have significantly reduced volumes in comparison to CKCSs without SM.

\section{Discussion}

The cranial base is of crucial importance in skull development. Unlike most of the skull bones, it is formed through endochondral ossification of its cartilaginous anlages. During the ontogenesis of the skull, ossification centres form within these cartilages and the segments separating these centres are referred to as synchondroses [26]. In the postnatal period, ongoing multiplication of chondrocytes and gradual ossification of the synchondroses contributes to the expansion of the cranial base analogous to the growth plates of long bones [11]. Any disruption of the growth of these synchondroses can lead to skeletal and craniofacial malformations such as achondroplasia. In achondroplastic animals and humans, the growth of all synchondroses is impaired to various degrees, resulting in reduced longitudinal extension of the cranial base as well as in the long bones. Brachycephaly is one consequence of this growth retardation [27-30].

The volume of the endocranial cavity is reduced less than one would expect from the degree of shortening of the neurocranium [16]. However, raised ICP has been found in achondroplastic patients. An association between an impaired outflow of blood at the exit foramina of the venous sinuses at the skull base has been proposed as the underlying etiology of this increased ICP, rather than an overall reduced intracranial volume [31]. The JF provides the extracranial passage for the sigmoid sinus, which is 
the major drain from the brain via the petrosal and transverse sinuses, flowing into the internal jugular vein $[32,33]$. Obstruction of the cerebral veins will increase ICP in more than one way. Holding of venous blood back in the skull, distention of the cerebral veins and venous hypertension follows. This in turn impairs cerebrospinal fluid (CSF) absorption at the arachnoid granulations, resulting in accumulation of CSF in the ventricles and communicating hydrocephalus $[31,34,35]$. Enlargement of the jugular foramen in this condition has successfully reduced intracranial pressure and hydrocephalus [36]. The venous congestion and elevated ICP have also been associated with turbulent flow and jets of CSF as well as with cerebellar herniation $[37,38]$. This might also play a role as a pathogenetic factor in the development of SM in CKCSs, as has been proposed previously [39].

In this study we focused on examination of the JF, because the other exit foramen for cerebral veins, the postglenoid foramen, has been found to be very variable. In canids the postglenoid foramen can even be divided so some dogs can have multiple postglenoid foramina. Its diameter is only $30 \%$ of the dimension of the JF in dogs. In contrast the JF is singular and characterized by minor variability in its morphology [25]. We use the term "jugular foramen" to describe the outflow tract of the intracranial veins for convenience. Actually, the JF is only the internal opening on the floor of the cranial cavity passing into a canal, whose extracranial opening is the tympano-occipital fissure (Figure 2). The JF is not easy to conceptualize due to its curved course. Measurements of the apical diameter of the JF in the two groups were attempted in this study, but minimal deviation of the image plane can lead to very variable diameters. For this reason volumes rather than diameters of the JF were determined, because diameters are strongly influenced by variations in orthograde positioning of the head. One limitation of our study was the exact ventral delineation of the JF, which had to be made by defined lines rather than by natural landmarks.

The results presented in this study can explain turbulent flow of CSF in the subarachnoid space as a consequence of raised ICP. This concept is independent of possible disproportionate volumes of the caudal skull compartment and the cerebellum. The increase in ICP could further exacerbate the CSF pulse pressure created by the decreased compliance in the subarachnoid space, which is considered to be the driving force of SM [5]. It can also explain why dogs with $\mathrm{CM} / \mathrm{SM}$ often experience rapid worsening of clinical signs during or immediately after exercise, after abdominal wall straining at defecation or after excitement [5]. In these conditions return of systemic blood to the heart is impeded by pressure inside the chest and abdomen, leading to backpressure in the venous system and a rise in ICP. This could contribute to an exaggerated systolic CSF pressure in the spinal compartment. However, it seems likely that multiple pathogenetic factors act together or sequentially in the development of SM in the CKCS.

Although the findings of this study seem to present a plausible explanation for an increase in subarachnoid CSF pressure, it must be emphasized that this study merely presents morphological findings and definite data about a functional impairment of venous outflow needs to be obtained. Angiographic presentation of a collateral network of emissary veins trying to compensate for the constrained venous drainage would give further evidence of an existing functional impairment of blood flow [40].

Sagittal sinus blood flow has been reported to be decreased in children with acondroplasia and constrained venous drainage from smaller JFs [41]. Determination of the blood flow in the sagittal sinus of the CKCS could also give further evidence of an existing functional impairment confirming our hypothesis from pure morphological data. Volume determination of the JF is a time consuming technique requiring specialized software and CT scanning of the dogs. However, quantification of intracranial venous blood flow might be a useful adjunct to the current imaging assessment of CKCS for breeding selection. Using MR-venography and cine phase contrast techniques, it could be possible to identify dogs having a higher risk of developing SM if impaired blood flow could be confirmed in follow-up studies.

Furthermore, treatment of raised ICP in these dogs might be directed at improving intracranial venous drainage rather than at vault expansion or CSF shunting procedures. Relief of raised ICP by surgical decompression of JF stenosis has been reported in children with achondroplasia and craniosynostosis [36], and ICP elevation might be amenable to surgical foraminoplasty in the CKCS, too.

\section{Conclusion}

A stenosis of the JF has been identified in CKCSs with $\mathrm{SM}$. A consecutive venous congestion may explain the aetiology of CSF pressure waves in the subarachnoid space independent from cerebellar herniation or as an additional pathogenetic factor for the development of SM in this breed.

\section{Abbreviations \\ CLM: Chiari-like malformation; CKCS: Cavalier King Charles Spaniel; \\ CSF: Cerebrospinal fluid; CT: Computed tomography; FLAIR: Fluid attenuated inversion recovery; FFE: Fast field echo; ICP: Intracranial pressure; JF: Jugular foramen; MR: Magnetic Resonance; SD: Standard deviation; \\ SM: Syringomyelia; 3D: Three-dimensional.}

\section{Competing interests}

The authors declare that they have no competing interests.

\section{Author contributions}

$\mathrm{HV}$ and MS conceived the study and planned the volume determinations, MK participated in the design and coordination of the study. NO performed 
all diagnostic imaging procedures and selected appropriate image sets. Maren Sauerbrey and CR performed the statistics. MS drafted the manuscript and $\mathrm{NO}$ and HV helped to draft the manuscript. All authors have approved the final manuscript.

\section{Author details}

${ }^{1}$ Department of Veterinary Clinical Sciences, Small Animal Clinic, Justus-Liebig-University, Frankfurter Straße 108, 35392 Giessen, Germany. ${ }^{2}$ Institute for Veterinary Physiology and Biochemistry, Justus-Liebig-University, Frankfurter Straße 100, 35392 Giessen, Germany. ${ }^{3}$ Department of Veterinary Clinical Sciences, Royal Veterinary College, Hawkshead Lane, Hatfield AL9 7TA, UK.

Received: 22 January 2012 Accepted: 28 July 2012

Published: 6 September 2012

\section{References}

1. Carrera I, Dennis R, Mellor DJ, Penderis J, Sullivan M: Use of magnetic resonance imaging for morphometric analysis of the caudal cranial fossa in Cavalier King Charles Spaniels. Am J Vet Res 2009, 70:340-345.

2. Cerda-Gonzalez S, Olby NJ, McCullough S, Pease AP, Broadstone R, Osborne J: Morphology of the caudal fossa in Cavalier King Charles Spaniels. Vet Radiol Ultrasound 2009, 50:37-46.

3. Cross HR, Capello R, Rusbridge C: Comparison of cerebral cranium volumes between Cavalier King Charles Spaniels with Chiari like malformation, small breed dogs and Labradors. J Small Anim Pract 2009, 50:399-405.

4. Lu D, Lamb CR, Pfeiffer DU, Targett M: Neurological signs and results of magnetic resonance imaging in 40 Cavalier King Charles Spaniels with Chiari type 1-like malformation. Vet Rec 2003, 153:260-263.

5. Rusbridge C, Greitz D, Iskandar J: Syringomyelia: current concepts in pathogenesis, diagnosis and treatment. J Vet Int Med 2006, 20:469-479

6. Schmidt MJ, Biel M, Klumpp S, Schneider M, Kramer M: Evaluation of the volumes of cranial cavities in Cavalier King Charles spaniels with Chiarilike malformation and other brachycephalic dogs as measured via computed tomography. Am J Vet Res 2009, 70:508-512.

7. Carruthers H, Rusbridge C, Dubé MP, Holmes M, Jeffery N: Association between cervical and intracranial dimensions and syringomyelia in the cavalier King Charles spaniel. J Small Anim Pract 2009, 50:394-398.

8. Tubbs RS, Elton S, Grabb P, Dockery SE, Bartolucci AA, Oakes WJ: Analysis of the posterior fossa in children with the Chiari 0 malformation. Neurosurgery 2001, 48:1050-1054.

9. Vega A, Quintana F, Berciano J: Basichondrocranium anomalies in adult Chiari type I malformation: a morphometric study. J Neurol Sci 1990, 99:137-145.

10. Sekula RF Jr, Jannetta PJ, Casey KF, Marchan EM, Sekula LK, McCrady CS: Dimensions of the posterior fossa in patients symptomatic for Chiari I malformation but without cerebellar tonsillar descent. Cerebrospinal Fluid Res 2005, 2:1-11.

11. Schmidt MJ, Neumann AC, Amort KH, Failing K, Kramer M: Cephalometric measurements and determination of the general skull type of Cavalier King Charles Spaniels. Vet Radiol Ultrasound 2011, 52:436-440.

12. Dewey CW, Berg JM, Barone G, Marino DJ, Stefanacci JD: Foramen magnum decompression for treatment of caudal occipital malformation syndrome in dogs. J Am Vet Med Assoc 2005, 227:1270-1275.

13. Rusbridge C, Knowler SP, Pieterse L, MC Fadyen AK: Chiari like malformation in the Griffon Bruxellois. J Small Anim Pract 2009, 50:386-393.

14. Dye JA, Kinder FS: A prepotent factor in the determination of skull shape. Am J Anat 1943, 54:333-346.

15. Montavon PM: Le syndrome brachycephalique. Proceedings, 1ère recontres Franco-Suisses. 2000.

16. Di Mario FJ, Ramsby GR, Burleson JA, Greensheilds IR: Brain morphometric analysis in achondroplasia. Neurology 1995, 45:519-524.

17. Gordon N: The neurological complications of achondroplasia. Brain Dev 2000, 22:3-7.

18. Fernández AA, Guerrero Al, Martínez MI, Vázquez ME, Fernández JB, Chesai Octavio E, Labrado Jde L, Silva ME, de Araoz MF, García-Ramos R, Ribes MG, Gómez C, Valdivia Jl, Valbuena RN, Ramón JR: Malformations of the craniocervical junction (Chiari type I and syringomyelia: classification, diagnosis and treatment). BMC Musculoskelet Disord 2009, 17;10(Suppl 1):1-11.
19. Erdinçler P, Dashti R, Kaynar MY, Canbaz B, Çiplak N, Kuday C: Hydrocephalus and chronically increased intracranial pressure in achondroplasia. Childs Nerv Syst 1997, 13:345-448.

20. Rich PM, Cox TCS, Hayward RD: The Jugular Foramen in Complex and Syndromic Craniosynostosis and Its Relationship to Raised Intracranial Pressure. AJNR Am J Neuroradiol 2003, 24:45-51.

21. Berlis A, Putz MD, Schumacher MD: Direct and CT measurements of the canals and foramina of the skull base. Brit J Radiol 1992, 65:653-661.

22. Onar V, Kahveciodlu KO, Cebi V: Computed tomographic analysis of the cranial cavity and neurocranium in the German shepherd dog (Alsatian) puppies. Veterinarski Arhiv 2002, 72:57-66.

23. Driver CJ, Rusbridge C, Cross HR, McGonnell I, Volk HA: Morphometric assessment of cranial volumes in age-matched Cavalier King Charles spaniels with and without syringomyelia. Vet Rec 2010, 167:978-979.

24. Parker JE, Knowler SP, Rusbridge C, Noorman E, Jeffery ND: Prevalence of asymptomatic syringomyelia in Cavalier King Charles spaniels. Vet Rec 2011, 168:667-669.

25. Wysocki J: Morphology of the temporal canal and postglenoid foramen with reference to the size of the jugular foramen in man and selected species of animals. Fol Morphol (Warsz) 2002, 61:199-208.

26. Evans HE: Miller's Anatomy of the Dog. 3rd edition. Totowa, New Jersey: WB. Saunders; 1993:122-166.

27. Grüneberg $\mathrm{H}$ : Systemic disorder of the cartilaginous skeleton. In The Pathology of Development. Blackwell: Blackwell, Chap; 1963:52-73. Chap. 3.

28. Almlöf J: On achondroplasia in the Dog. Zentralb/ Veterinärmed 1961, 8:43-56.

29. Braund KG, Ghosh P, Taylor TK, Larsen LH: Morphological studies of the canine intervertebral disc. The assignment of the beagle to the achondroplastic classification. Res Vet Sci 1975, 19:167-172.

30. Jezyk PF: Constitutional Disorders of the Skeleton in Dogs and Cats. In Textbook of Small Animal Orthopaedics. Edited by Newton CD, Nunamaker DM. Ithaca, New York, USA: Publisher: International Veterinary Information Service (www.ivis.org); 1985

31. Cinalli G, Saint-Rose C, Kollar EM: Hydrocephalus and craniosynostosis. J Neurosurg 1995, 88:209-214.

32. Butler $\mathrm{H}$ : The development of mammalian dural venous sinuses with special reference to the post-glenoid foramen. J Anat 1967, 102:33-52.

33. Reinhard KR, Miller ME, Evans HE: The craniovertebral veins and sinuses of the dog. Am J Anat 1992, 111:67-87.

34. Taylor WJ, Hayward RD, Lasjaunias P, Britto JA, Thompson DN, Jones BM, Evans RD: Enigma of raised intracranial pressure in patients with complex craniosynostosis: the role of abnormal intracranial venous drainage. J Neurosurg 2001, 94:377-385.

35. Moritani T, Aiharaa T, Ogumaa E, Makiyamab Y, Nishimotob H, Smokerc WRK, Satoc Y: Magnetic resonance venography of achondroplasia: correlation of venous narrowing at the jugular foramen with hydrocephalus. Clin Imag 2006, 30:195-200.

36. Lundar T, Bakke SJ, Nornes H: Hydrocephalus in an achondroplastic child treated by venous decompression at the jugular foramen. J Neurosurg 1990, 73:138-140.

37. Thompson DNP, Harkness W, Jones BM, Hayward RD: Aetiology of herniation of the hindbrain in craniosynostosis. Ped Neurosurg 1997, 26:288-295

38. Cinalli G, Spennato P, Sainte-Rose C, Arnaud E, Aliberti F, Brunelle F, Cianciulli E, Renier D: Chiari malformation in craniosynostosis. Childs Nerv Syst 2005, 21:889-901.

39. Scrivani PV, Freer SR, Dewey CW, Cerda-Gonzalez S: Cerebrospinal fluid signal void sign in dogs. Vet Radiol Ultrasound 2009, 50:269-275.

40. Rollins N, Booth T, Shapiro K. The use of gated cine phase contrast and MR venography in achondroplasia. Childs Nerv Syst 2000, 16:569-577.

41. Hirabuki N, Watanabe Y, Mano T, Manoa T, Fujitaa N, Tanakaa H, Ueguchia T, Nakamuraa H: Quantification of flow in the superior sagittal sinus performed with cine phase-contrast MR imaging of healthy and achondroplastic children. AJNR Am J Neuroradiol 2000, 21:1497-1501.

doi:10.1186/1746-6148-8-158

Cite this article as: Schmidt et al.: Volume reduction of the jugular foramina in Cavalier King Charles Spaniels with syringomyelia. BMC Veterinary Research 2012 8:158. 